Published in final edited form as:

Science. 2015 March 06; 347(6226): 1069-1070. doi:10.1126/science.1252967.

\title{
Drugging RNAi:
}

RNAi therapeutics are emerging as a major drug discovery engine

\author{
Dirk Haussecker ${ }^{1}$ and Mark A. Kay ${ }^{2}$ \\ ${ }^{1}$ RNAi Therapeutics Consulting, Rastatt, Germany \\ ${ }^{2}$ Pediatrics and Genetics, Stanford University, Stanford, CA, USA
}

RNA interference (RNAi)-based drugs harness endogenous posttranscriptional gene silencing pathways for therapeutic purposes. The goal is to turn down or shut off the expression of genes known to contribute to or cause disease. RNAi "triggers" are typically double-stranded RNAs (dsRNAs) of which one strand has a sequence complementary to that of a messenger RNA (mRNA), resulting in the reduction or elimination of that an mRNA and its corresponding protein product. The dsRNAs can be provided as synthetic oligonucleotides or as genetic DNA templates from which the RNAi triggers are transcribed in the target cells (vector-based transcriptional RNAi) (see the figure).

Key to the therapeutic utility of these RNAi triggers is the ability to introduce them into their target cells in the body. Such delivery is typically facilitated by formulation into nanoparticles, simple conjugates, or viral vectors (see the figure). To date, at least three delivery technologies (liposomal nanoparticles, simple conjugates, and polyconjugates) have shown highly persistent silencing of target gene expession in the liver of humans and nonhuman primates, suggesting therapeutic dosing frequencies as low as once-monthly or once-quarterly (1-3).

There are two lead RNAi drug candidates (ALN-TTR02 and ALN-TTRsc) in phase III trials that target the disease-causing mutant transthyretin (TTR) mRNA in the liver for the treatment of familial amyloid polyneuropathy. Given that deficiency of the TTR gene product is expected to be well tolerated and the mutant TTR protein causes the disease, the target risk is low, and commercialization may happen as early as 2017 (ALN-TTR02). Beyond the TTR amyloidosis candidates, there is an expanding pipeline of RNAi gene targets in the liver. These include candidates for diseases ranging from important public health issues (e.g., hepatitis B virus infection, common forms of metabolic and cardiovascular disorders, liver cancer) to the rare and severe (e.g., triglyceride-related pancreatitis, primary hyperoxaluria $1, a_{1}$-antitrypsin-related liver disease). $N$-Acetylgalactosamine (GalNAc) siRNA conjugates targeting the liver have emerged as an attractive delivery option offering the prospect of infrequent (once-monthly or even once-quarterly) subcutaneous dosing, making them suitable for other common chronic diseases such as type II diabetes and hypercholesterolemia (2).

Although the liver is a favored organ for delivery owing to its physiological role in removing particles from circulation, it is less clear whether new approaches aimed at nonhepatic tissues will provide therapeutic efficacy. These smaller nanoparticles, conjugates, self- 
delivering RNAi triggers, cationic lipoplexes, and transcriptional RNAi methods hold particular promise for targeting cancer cells, phagocytic cells, vascular endothelial cells, cell populations in the kidney, cells in the back of the eye, and the various cells types in the central nervous system (CNS) (4).

For diseases requiring life-long treatment, as well as for the hard-to-reach (e.g., CNS) tissues and/or tissues that rapidly turn over, such as blood-derived stem cells, transcriptional RNAi methods currently have a practical advantage, because of the prospect of persistent activity after single administration. In addition, transcriptional RNAi may be a better match for certain diseases where both the addition of a normal gene, as well as silencing of the endogenous mutated gene, are beneficial. This would include diseases such as sickle cell anemia (5) or the most common form of $a_{1}$-antitrypsin deficiency (6). However, one disadvantage is that dosing is more difficult to control with vector-transcribed RNAi. Transcriptional RNAi candidates in clinical development today address cancer, HIV, and hepatitis $\mathrm{C}$ virus, as candidates for $\mathrm{a}_{1}$-antitrypsin deficiency and neurodegenerative disorders approach the clinic. This compares to over 20 synthetic RNAi trigger clinical candidates.

It remains to be seen how the safety profile from the largely short-term experience ( 6 to 12 months) with the leading TTR amyloidosis candidates will hold up in the long run. So far, safety issues for the three most advanced delivery technologies (SNALP liposomal nanoparticles by Tekmira, GalNAc conjugates by Alnylam, and DPC polyconjugates by Arrowhead Research) have largely been related to the route of administration (infusion and injection site reactions) and the delivery chemistry, and not the RNAi mechanism of action per se $(1,2)$. In particular, the propensity of the larger nanoparticulate formulations such as liposomes to interact with the innate immune system has been a major challenge (7) and necessitated the adoption of transient immune suppression around the time of dosing. By contrast, smaller systems such as the DPCs and Gal-NAc conjugates rely on the use of heavily modified RNAi triggers that may accumulate in compartments such as lysosomes, the long-term safety of which remains to be determined.

Off-target activities, a challenge shared with other drug modalities, may also influence the long-term safety experience, especially because RNAi triggers may modulate expression of dozens of off-target genes (8). Minimizing off-target effects at the RNAi trigger design phase relies on error-prone bioinformatic filters rather than on the iterative chemical optimization and monitoring of off-target activities in preclinical models (9).

Another long-term safety issue is whether hijacking the endogenous microRNA pathway is safe given the functions of micro-RNAs throughout biology. Studies involving the use of RNAi triggers generated from strong transcriptional promoters revealed that competition between therapeutic RNAi triggers and endogenous microRNAs for the same machinery can have adverse consequences given the pervasive importance of microRNAs in gene regulation (10). Nevertheless, improvements in RNAi trigger design to facilitate more efficient processing should lessen the burden by avoiding congestion of the RNAi pathway (11). 
The history of investment in RNAi therapeutics has been something of a roller coaster ride. Initial interest in the therapeutic application of RNAi was limited to small biotechnology companies specialized in oligonucleotide therapeutics development (2002 to 2005). There followed a sudden stampede into the the technology by the pharmaceutical industry (12). This period of irrational exuberance (2005 to 2008) was preceded by initially measured investments by some large companies (Medtronic, Merck, Novartis) and the award of the Nobel Prize for the discovery of dsRNA as the efficient trigger of the RNAi gene silencing process. This exuberance culminated in bidding wars, unrealistic expectations, and misplaced investments, precipitating a considerable backlash (2008 to 2011). It was uncertain whether the industry could ever recover (12). The realization that many of the early most prominent claims for therapeutic gene silencing in animal models of human disease were in retrospect best explained by innate immunostimulatory, antiproliferative, and antiviral artifacts was a further cause of uncertainty. Important scientific breakthroughs at this time, such as the demonstration of on-target RNAi in nonhuman primates, advances in conjugate delivery, and strategies to abrogating innate immune stimulation, struggled to be appropriately recognized (12). It was only after a string of demonstrations of target gene silencing in humans that the industry started to recover (13).

There has also been competition from other nucleic acid therapeutics platforms, in particular ribonuclease $\mathrm{H}$ (RNase $\mathrm{H}$ ) antisense technology and genome editing. Especially with recent advances in ligand-targeted delivery, the long-held potency advantage of RNAi has diminished relative to RNase $\mathrm{H}$ antisense technology (14). But competition, both among RNAi technologies and with antisense oligonucleotides, has also driven much progress in the field. With more than $\$ 1$ billion invested in RNAi-based therapeutics in the first 6 months of 2014 alone (13), together with the increased acceptance of gene therapy in general, there should be a rapidly expanding clinical pipeline of RNAi-based drugs and a maturing of delivery technologies for targeting cells and tissues outside the liver.

\section{References}

1. Coelho T, et al. N Engl J Med. 2013; 369:819. [PubMed: 23984729]

2. www.alnylam.com/web/assets/MASTER-RD-DAY-DECK_Capella.pdf

3. Wooddell CI, et al. Mol Ther. 2013; 21:973. [PubMed: 23439496]

4. Haussecker D. J Control Release. 2014; 195:49. [PubMed: 25111131]

5. Samakoglu S, et al. Nat Biotechnol. 2006; 24:89. [PubMed: 16378095]

6. Mueller C, et al. Mol Ther. 2012; 20:590. [PubMed: 22252449]

7. http://investor.tekmirapharm.com/releasedetail.cfm?ReleaseID $=857714$

8. Jackson AL, et al. RNA. 2006; 12:1179. [PubMed: 16682560]

9. Vaish N, et al. Nucleic Acids Res. 2011; 39:1823. [PubMed: 21047800]

10. Grimm D, et al. Nature. 2006; 441:537. [PubMed: 16724069]

11. Gu S, et al. Cell. 2012; 151:900. [PubMed: 23141545]

12. Haussecker D. Mol Ther Nucleic Acids. 2012; 1:e8. [PubMed: 23344723]

13. Maraganore J. Nature. 2014; 510:35.

14. Prakash TP, et al. Nucleic Acids Res. 2014; 42:8796. [PubMed: 24992960] 


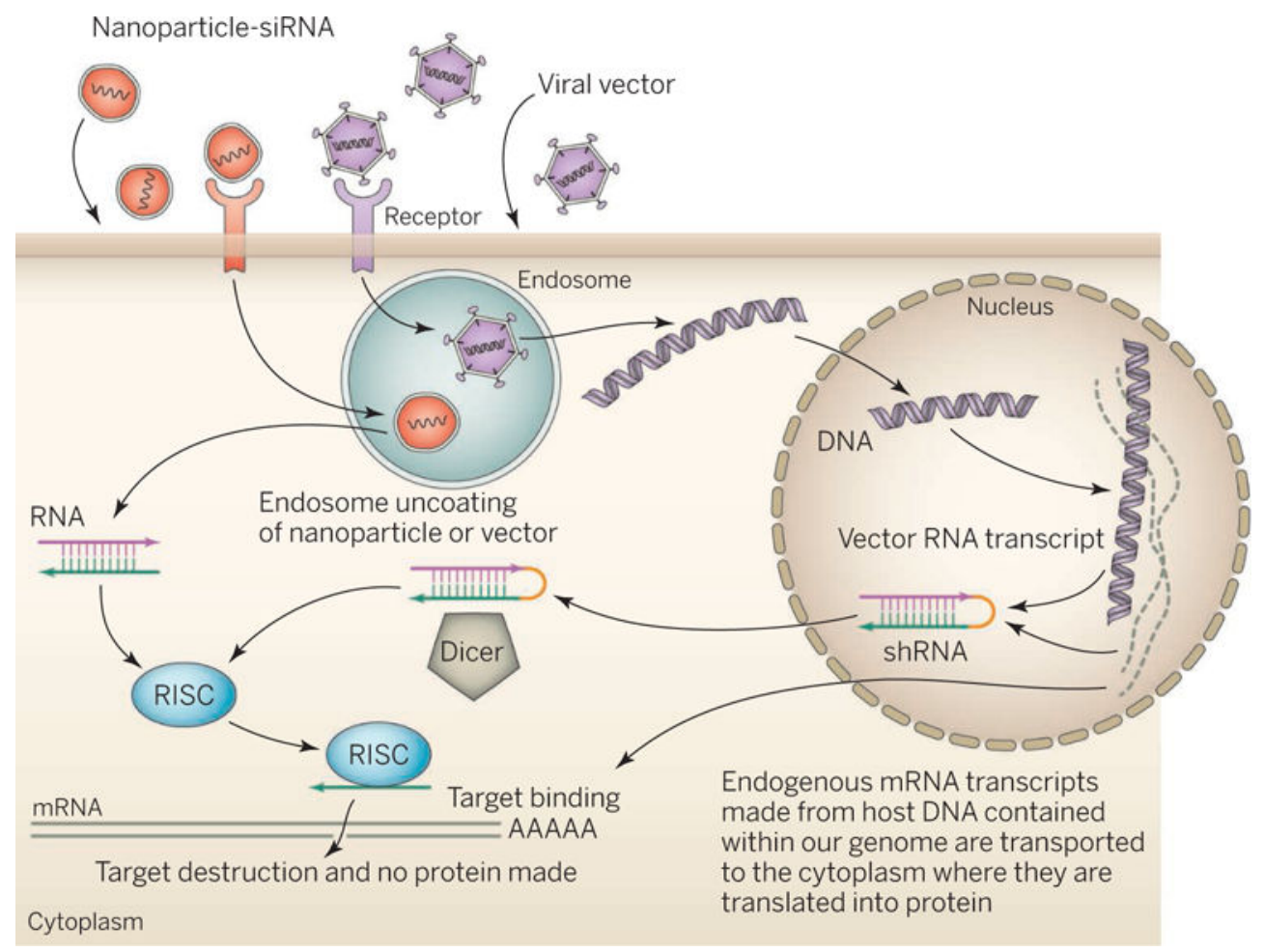

Figure. RNAi is the drug

RNAi delivery approaches include conjugates, liposomal nanoparticles (LNPs), and viral vectors. Nanoparticles deliver synthetic dsRNA, whereas viral vectors deliver a transcriptional template to the nucleus. Cellular uptake occurs nonspecifically or via receptor-mediated endocytosis. Nanoparticles deliver the RNAi trigger to the cytoplasm. The trigger enters the RNAi pathway at the RISC or Dicer processing stage. Transcriptional templates produce hairpin RNAs that enter the pathway at an earlier, nuclear stage. Ultimately, an active RISC complex is formed that cleaves the mRNA target. RISC, RNAinduced silencing complex; shRNA, short hairpin-mediated RNA. 